\title{
Crystallization and preliminary $X$-ray analysis of bacteriophage lambda lysozyme in which all tryptophans have been replaced by aza-tryptophans
}

\author{
Christine Evrard, ${ }^{a, b}$ JeAn-Paul DeClercQ ${ }^{a}$ and Jac'Ques Fastrez ${ }^{b}$ at ${ }^{a}$ Laboratoire de Chimie Physique et de \\ Cristallographie, Université Catholique de Louvain. 1 Place Louis Pasteur, B-1348, Louvain-La-Neuve. Belgium, and ${ }^{h}$ Laboratoire \\ de Biochimie Physique et des Biopolvmères, Université Catholique de Louvain, 1 Place Louis Pasteur, B-1348, Louvain-La-Neuve, \\ Belgium. E-mail: delercq(a)cpmc.ucl.ac.be
}

(Received 8 March 1996; accepted 17 September 1996)

\begin{abstract}
After many unsuccessful attempts to crystallize the bacteriophage lambda lysozyme, a mutant where all the tryptophan residues have been replaced by aza-tryptophans has been crystallized by the vapor-diffusion method. The crystals are orthorhombic and belong to space group $P 2_{1} 2_{1} 2_{1}$ with cell dimensions $a=73.01, b=78.80, c=82.31 \AA$. Diffraction data were collected using synchrotron radiation sources. Crystals diffract to a resolution of $2.3 \AA$. Data from two different platinum derivatives were also recorded to 2.8 and $2.5 \AA$, respectively.
\end{abstract}

\section{Introduction}

The bacteriophage lambda lysozyme $(\dot{i L})$ is a small protein of 158 amino acids. Like other known lysozymes, it is involved in the lysis of the cell walls of bacteria. Lysozymes are widespread in nature and have been isolated from a variety of organisms. Several classes of lysozymes have been defined on the basis of amino-acid sequence similarities. The crystal structures of lysozymes belonging to three different classes are known: type $C$ (chicken), type $G$ (goose) and type V (viral). No sequence homology is detected in lysozymes of different classes but nevertheless structural similarities are observed between them, suggesting the hypothesis of a diverging evolution from a remote common ancestor (Rossmann \& Argos, 1976). In the three classes, the amino terminal contains the catalytic residues and consists of an $\alpha$-helix followed by a three-stranded $\beta$-sheet. A precise structural alignment has been performed by Taylor $\&$ Orengo (1989): the alignment of residues in the $\beta$-sheets of $T_{4} L$ (bacteriophage $\mathrm{T}_{4}$ lysozyme) and HEWL (hen egg-white lysozyme) is given in Fig. 1.

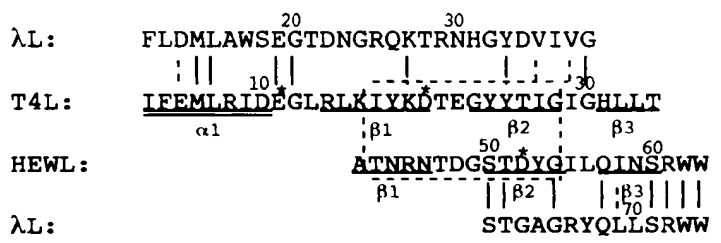

Fig. 1. Multiple alignment of the amino-terminal part of the sequence of $i \mathrm{~L}$ with the sequence of $\mathrm{T}_{4} \mathrm{~L}$ and HEWL. The symbols $\mid$ and indicate the presence of identical or similar residues in the homologous fragments. The two boxed sequences are aligned on the basis of structural homology as described by Taylor \& Orengo (1989). The $\alpha$-helixes and the $\beta$-strands of $T_{4} L$ and HEWL are doubly or singly underlined. The catalytic residues of $\mathrm{T}_{4} \mathrm{~L}$ and HEWL are indicated by an asterisk.
An evolutionary relationship between $\lambda \mathrm{L}$ and the $\mathrm{V}$ - and C-type lysozymes has been discussed in detail by Jespers, Sonveaux \& Fastrez (1992) who aligned the amino-terminal sequences of $i \mathrm{~L}$ and $\mathrm{T}_{4} \mathrm{~L}$. This local alignment encompasses the first helix of $\mathrm{T}_{4} \mathrm{~L}$ and most of the $\beta$-sheet, suggesting a critical catalytic role for Glu 19 of $\lambda \mathrm{L}$. The inactivation of the enzyme by mutation of Glul9 into Gln supports this hypothesis. The C-terminal sequences of $\lambda \mathrm{L}$ and of $\mathrm{T}_{4} \mathrm{~L}$ can also be aligned. These observations indicate that $\dot{\lambda} \mathrm{L}$ is related to the V-type lysozymes. In attempts to align the sequence of $\lambda \mathrm{L}$ with those of the C-type lysozymes, a fragment of 29 residues showing significant similarity was detected; it is located in the middle of the sequence of $\lambda \mathrm{L}$ and aligns with the $\beta$-sheet part of the C-type lysozymes. Consequently, two regions in the sequence of $\lambda \mathrm{L}$ align with a $\beta$-sheet structure if one considers, on the one hand, the alignment with $\mathrm{T}_{4} \mathrm{~L}$ and, on the other hand, the alignment with HEWL. These alignments suggest that there is a repetition of this motif in iL (Fig. 1). The distance between the two fragments (29 amino acids) coincides with the length of the high score alignment observed with C-type lysozymes.

All these observations suggest that $\lambda \mathrm{L}$ could represent an evolutionary branch between C-type and V-type lysozymes, although the mechanism of action of $\lambda \mathrm{L}$ differs. Lysozymes cleave the glycosidic bond between $\mathrm{Cl}$ of $\mathrm{N}$-acetyl muramic acid (NAM) and $\mathrm{C} 4$ of $N$-acetyl glucosamine (NAG) of the peptidoglican of the bacterial wall. The mechanism of HEWL, GEWL (goose egg-white lysozyme) and $\mathrm{T}_{4} \mathrm{~L}$ is hydrolytic, while $i \mathrm{~L}$ acts as a transglycosidase (Taylor, Das \& van Heijenoort, 1975).

The determination of the three-dimensional structure of $\lambda \mathrm{L}$ should be helpful in elucidating the difference in its enzymatic mechanism in comparison with other classes of lysozymes. It would also provide very useful information, regarding the mechanism of evolution of proteins.

\section{Experimental}

The bacteriophage lambda lysozyme was overexpressed in Escherichia coli and purified by a modification of a previously published protocol that included DEAE-cellulose, S-Sepharose Fast Flow and Sephadex G75 steps (Jespers, 1991; Jespers, Sonveaux, Fastrez, Phanapoulos \& Davison, 1991: Soumillion $\&$ Fastrez, 1992). The only modification concerns the use of a phosphate buffer during the S-Sepharose Fast Flow step instead of a Hepes buffer. The purity of the enzyme (99\%) was estimated by sodium dodecylsulfate polyacrylamide gel electrophoresis (SDS-PAGE) and analytical chromatography on Pharmacia Mono S column. The lysozyme was concentrated by ultrafiltration (Millipore immersible $\mathrm{CX}-10$ and Ultrafree-PF) to $20 \mathrm{mg} \mathrm{ml}^{-1}$ in $50 \mathrm{mM} \mathrm{NaH} \mathrm{PO}_{4} / \mathrm{Na}_{2} \mathrm{HPO}_{4}$ pH 6.7, $\mathrm{NaN}_{3}$ 
$0.02 \%$. The final concentration was determined from absorbance at $280 \mathrm{~nm}$ using the molar absorption coefficient given by Soumillion, Jespers, Vervoort \& Fastrez (1995).

In spite of numerous attempts, we were unable to grow crystals suitable for X-ray analysis from the native enzyme. A number of mutants were then used for screening the crystallization conditions (Jancarik \& Kim, 1991) and the best results were obtained with a molecule in which the four tryptophan residues were replaced by aza-tryptophans (I) according to the experimental procedure described by Soumillion et al. (1995). The purification and concentration of this mutant follows the same protocol as the wild type.

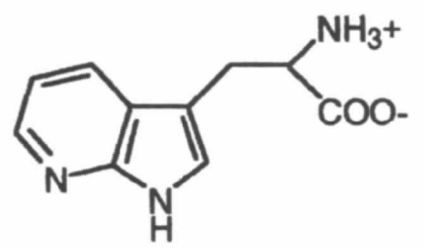

(I)

This mutant was selected by NASA (National Aeronautics and Space Administration) for crystallization experiments under microgravity conditions during the Space Shuttle flights STS-63 and STS-67. The PCAM (Protein Crystallization Apparatus for Microgravity) experimental hardware (sitting-drop vapour diffusion) was used. Crystals were grown at $291 \mathrm{~K}$ : $5 \mu \mathrm{l}$ of the protein solution $\left(20 \mathrm{mg} \mathrm{ml}^{-1}\right.$ in $50 \mathrm{mMNaH} \mathrm{NaO}_{4} / \mathrm{Na}_{2} \mathrm{HPO}_{4}$ $\mathrm{pH} 6.7, \mathrm{NaN}_{3} 0.02 \%$ ) was mixed with an aliquot of $5 \mu \mathrm{l}$ of the reservoir solution [total volume $500 \mu \mathrm{l}: 0.1 \mathrm{M}$ sodium citrate $\mathrm{pH}$ $5.3,15 \%(v / v)$ 2-propanol and $20 \%(w / v)$ polyethylene glycol $4000]$. Needles of typical dimensions $0.10 \times 0.12 \times 1.00 \mathrm{~mm}$ (Fig. 2) were obtained at the end of flight STS-67 (16 d). Precession photographs using a rotating-anode generator showed that the crystals are orthorhombic, space group $P 2_{1} 2_{1} 2_{1}$ with unit-cell dimensions $a=73.01, \quad b=78.80$, $c=82.31 \AA$ (values refined by the program SCALEPACK at the end of the data collection). Data were collected at room temperature using a synchrotron radiation source at the EMBL Hamburg outstation. Crystals diffracted to a resolution of $2.3 \AA$ on the X31 beamline $(\lambda=1.000 \AA)$, using a MAR Research imaging-plate detector (Fig. 3). Data were processed using DENZO (Otwinowski, 1993) then scaled by SCALEPACK (Otwinowski, 1993). The scale and the temperature factors between the successive images remained essentially constant, showing a total absence of crystal decay in the synchrotron $\mathrm{X}$-ray beam during the time necessary for data collection. A total of 104257 reflections were recorded among which 21455 independent reflections with an $R_{\text {merge }}(I)$ of $9.0 \%(98.8 \%$ completeness). It is likely that the relatively high value of this index may be related to the high degree of redundancy (about 5) of the data. Average intensities in the highest resolution shell $(2.34-2.30 \AA)$ are more than three times their standard deviation.

\section{Discussion}

If we assume that two or three molecules are present in the asymmetric unit, the calculation of $V_{M}$ (Matthews, 1968) leads to values of 3.4 and $2.2 \AA^{3} \mathrm{Da}^{-1}$, respectively, with associated solvent content of 62 and $44 \%$. Both $V_{M}$ values are consistent with those obtained for globular proteins though the situation in which the asymmetric unit contains three molecules seems more probable. In order to clarify the ambiguity, self-rotation function searches were attempted looking for non-crystallographic symmetry (program $X-P L O R$, Brünger, 1992). A systematic search through all rotation possibilities was conducted using several ranges of resolution. It was expected to find a twofold or threefold rotation axis but none of them was observed; in fact the self-rotation function was completely flat and no conclusive result was obtained. Moreover, no pure translation peak was found in the Patterson function and the unit-cell content remains undefined up to now.

As explained in the Introduction, the sequence of $\lambda \mathrm{L}$ presents some similarities with those of $\mathrm{T}_{4} \mathrm{~L}$ and HEWL. Among them, $\mathrm{T}_{4} \mathrm{~L}$ presents the best percentage of amino-acid sequence

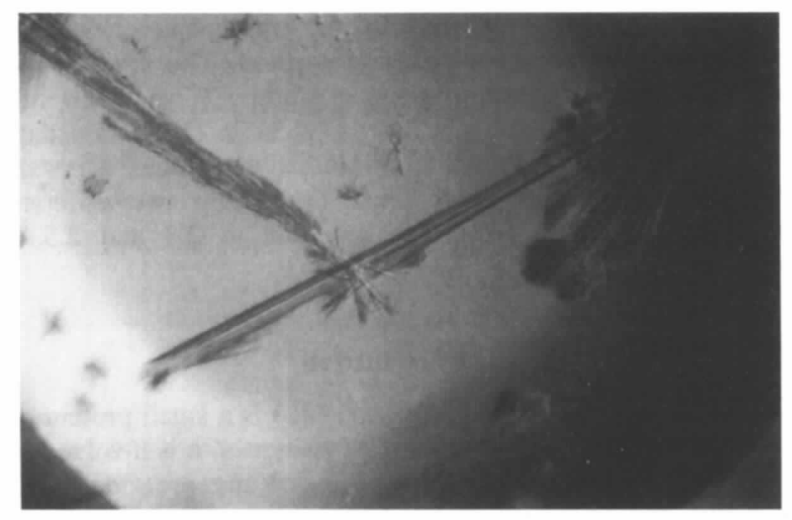

Fig. 2. Crystals of the aza-tryptophan mutant of bacteriophage lambda lysozyme grown in microgravity conditions during the Space Shuttle flight STS-67. The length of the largest needle is about $2 \mathrm{~mm}$.

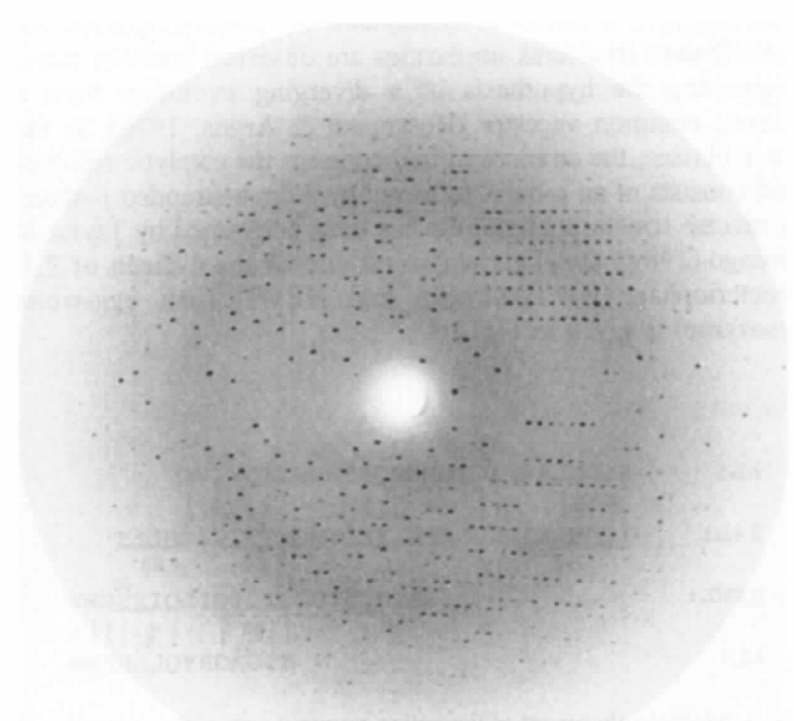

Fig. 3. A rotation image from a crystal of the aza-tryptophan mutant of bacteriophage lambda lysozyme recorded on a MAR Research imaging plate using the $\mathrm{X} 31$ beamline at EMBL Hamburg outstation. Rotation range $1.5^{\circ}$, crystal-to-film distance $190 \mathrm{~mm}$, exposure time $360 \mathrm{~s}$. The resolution at the edge of the photograph is $2.28 \AA$. 
similarity but it is only $18 \%$. Despite these low values the considerations presented in the Introduction suggest that it could eventually be possible to solve the phases problem by the molecular replacement method. According to Thunnissen et al. (1994), the C-terminal domain of the soluble lytic transglycosidase (SLT) from $E$. coli shows a folding very similar to those of HEWL and $T_{4} \mathrm{~L}$ and this part of the enzyme was also considered as a possible model for molecular replacement. All the attempts were performed using X-PLOR (Brünger, 1992), $A M o R e$ (Navaza, 1994), POLARRFN and $A L M N$, the latter two being part of the $C C P 4$ suite of programs (Collaborative Computational Project, Number 4, 1994). The coordinates of the lysozyme models were obtained from the Protein Data Bank (Bernstein et al., 1977): entries 3LZM ( $\left.\mathrm{T}_{4} \mathrm{~L}\right)$ and $2 \mathrm{LZT}$ (IEWL), those of SLT were kindly provided by the authors. We tried intensively to use the three full models as well as partial models from the two lysozyme molecules and also several combination of fragments, based on their sequence similarity with $i$ L. Several resolution ranges were tested but all these trials were unsuccessful, even for the cross-rotation part of the problem. This fact strengthens the assumption that the structure of $\lambda \mathrm{L}$ would be quite different from those of other classes of lysozymes and that the solution will require multiple isomorphous replacement.

Two platinum derivatives were prepared by soaking the crystals in the mother liquor with the addition of either $5 \mathrm{~m} M$ sodium tetrachloroplatinate $(2 \mathrm{~d})$ or $1 \mathrm{~m} M$ platinum terpyridine chloride (2-5 d). Derivative data including anomalous dispersion were collected to a resolution of 2.8 and $2.5 \AA$, respectively, on the wiggler beamline $\mathrm{BW} 7 \mathrm{~B}(\hat{\lambda}=0.859 \AA)$ at the EMBL Hamburg outstation. A total of 22714 independent reflections out of 60359 reflections were collected for the first derivative with an $R_{\text {merge }}(I)$ value of $7.4 \% \quad(98.9 \%$ of completeness) and 31791 independent reflections out of 77184 reflections for the second derivative with an $R_{\text {merge }}(I)$ value of $10.2 \%$ (98.9\% completeness). The scaling $R$ factors between the native and the two derivative crystals are 25.4 and $21.6 \%$, respectively. The analysis of these derivatives is now under way.

The authors are indebted to the Fonds National de la Recherche Scientifique (Belgium) and to the Fonds de
Développement Scientifique (Université Catholique de Louvain) for financial support. CE thanks the Fonds pour la Formation à la Recherche dans l'Industrie et dans l'Agriculture for a fellowship. We thank the European Union for support of the work at EMBL Hamburg through the HCMP to Large Installations Project, contract No. CHGE-CT93-0040. We are grateful to the members of the scientific staff at the EMBL outstation for their help during data collection and processing. The opportunity provided by NASA and in particular by $\mathrm{Dr}$ Daniel Carter for growing crystals in microgravity conditions is gratefully acknowledged. Finally, we would like to thank Michèle Bouchet for technical assistance.

\section{References}

Bernstein, F. C., Koetzle, T. F., Williams, G. J. B., Meyer, E. F. Jr, Brice, M. D., Rodgers, J. R., Kennard, O., Shimanouchi, T. \& Tasumi, M. (1977). J. Mol. Biol. 112, 535-542.

Brünger, A. T. (1992). X-PLOR Version 3.1, A System for X-ray Crnstallography and NMR. New Haven: Yale University Press.

Collaborative Computational Project, Number 4, (1994). Acta Cryst. D50, 760-763.

Jancarik, J. \& Kim, S.-H. (1991). J. Appl. Crist. 24, 409-411.

Jespers, L. (1991). PhD thesis, University of Louvain, Belgium.

Jespers, L., Sonveaux, E. \& Fastrez, J. (1992). J. Mol. Biol. 228, 529538.

Jespers, L., Sonveaux, E., Fastrez, J., Phanapoulos, A. \& Davison, J. (1991). Protein Eng. 4, 485-492.

Matthews, B. W. (1968). J. Mol. Biol. 33, 491-497.

Navaza, J. (1994). Acta Crnst. A50, 157-163.

Otwinowski, Z. (1993). Data Collection and Processing. Proceedings of the CCP4 Study Weekend, pp 56-62. Warrington, England: Daresbury Laboratory.

Rossmann, M. G. \& Argos, P. (1976). J. Mol. Biol. 105, 75-95

Soumillion, P. \& Fastrez, J. (1992). J. Biochem. 286, 187-191

Soumillion, P., Jespers, L., Vervoort, J. \& Fastrez, J. (1995). Protein Eng. 5, 451456.

Taylor, A., Das, B. C. \& van Heijenoort, J. (1975). Eur. J. Biochem. 53. 47-54.

Taylor, W. R. \& Orengo, C. A. (1989). J. Mol. Biol. 208, 1-22.

Thunnissen, A. W. H., Dijkstra, A. J., Kalk, K. H., Rozcboom, H. J., Engel, H., Keck, W. \& Dijkstra, B. W. (1994). Nature (London), 367, $750-753$. 